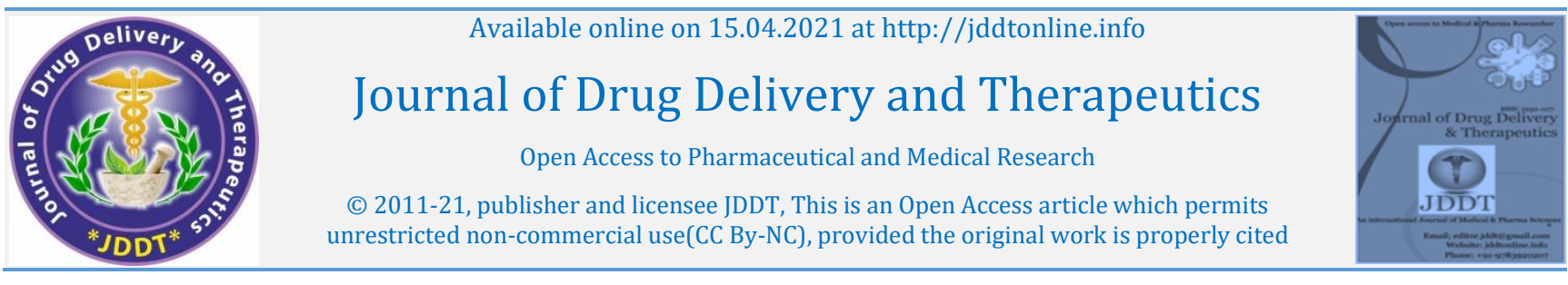

Open Access Full Text Article

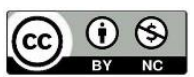

Review Paper

\title{
Mucoadhesive Microspheres: A Targeted Drug Delivery System
}

\author{
Hans Raj, Ankita Sharma, Shagun Sharma, Kapil Kumar Verma, Amit Chaudhary* \\ School of Pharmacy, Abhilashi University, Mandi-175028, Himachal Pradesh, India
}

\begin{tabular}{ll}
\hline Article Info: & \\
& Article History: \\
& Received 07 Feb 2021 \\
& Accepted 23 March 2021 \\
&
\end{tabular}

Cite this article as:

Raj H, Sharma A, Sharma S, Verma KK, Chaudhary A, Mucoadhesive Microspheres: A Targeted Drug Delivery System, Journal of Drug Delivery and Therapeutics. 2021; 11(2-s):150-155

DOI: http://dx.doi.org/10.22270/jddt.v11i2-s.4791

*Address for Correspondence:

Amit Chaudhary, School of Pharmacy, Abhilash

University, Mandi-175028, Himachal Pradesh, India

\section{Abstract}

Carrier technology is a novel and groundbreaking drug delivery system. Microspheres transmit the drug by affixing it to carrier particle-like Microspheres, Nanoparticles and Liposomes due to their smaller size and other useful properties. Mucoadhesive Microspheres are an integral component of this multi-particle drug delivery system and play an important function in the delivery of a novel drug. This drug delivery improves the therapeutic effectiveness of the drug. Mucoadhesive microspheres have a longer duration of residence at the absorption site, which leads to the accuracy of the drug targeting at the absorption site and improves the therapeutic efficacy of the drug. Mucoadhesive microspheres are formed by either systemic or local effects in gastrointestinal, oral, vaginal, nasal, rectal, ocular delivery. This is the ideal targeting device which could be done in a variety of ways.

Keywords: Microspheres, Bioadhesion, Polymer, Bioavailability

\section{INTRODUCTION}

Orally controlled release systems remain the most prominent among all drug delivery systems. This has many advantages compared to conventional systems, such as better plasma level profile, lower dosing, and toxicity, etc. ${ }^{1}$ Drug delivery systems (DDS) that can precisely control release rates or target drugs to particular body locations have had a profound effect on the healthcare system. Throughout treatment, the optimal drug delivery system delivers drugs at a pace determined by the body's needs and delivers the active entity specifically to the site of action. Through the coupling of drugs to carriers such as Microspheres, Nanoparticles and Liposome, amongst others, the carrier technology ensures an intelligent approach in drug delivery to modulate the release and the absorption of the drug. ${ }^{2}$ Many approaches are taken with a sustainable controlled release to deliver a drug to the target site. ${ }^{3}$ The most convenient and preferred mode of distribution to the body's systemic circulation is the oral administration of medicinal products. However, due to their inability to restrain and localize the system at the gastrointestinal tract, oral administration of most medications in conventional dosage forms has a short-term limitation. ${ }^{4}$ Microspheres are a type of novel drug delivery system comprised of different polymers and also have a variety of applications. ${ }^{5}$ For many years, microsphere carrier systems produced from naturally occurring biodegradable polymers have sparked significance in the development of sustained drug delivery. Recently, dosing forms which can precisely control release rates and target drugs to a specific body site have had an important impact on the formulation, production and delivery of new drug delivery systems. ${ }^{1}$ Microspheres are a vital aspect of this novel drug delivery system. Microspheres are a carrierlinked drug delivery mechanism with a drug core and outer layers of a polymer as a coating material, with particle sizes ranging from $1-1000 \mu \mathrm{m}$ in diameter. Due to their short duration of residence at the absorption site, the output of these microspheres is limited. It would be beneficial to provide a method for ensuring that the drug delivery mechanism is in close proximity to the absorbing membrane. ${ }^{5}$ The development of an oral controlled release Mucoadhesive multiparticulate device is one of many methods being explored to extend the gastric residence time of the dosage form at the absorption site. 1 This can be accomplished by combining bioadhesion properties with microspheres to create "Mucoadhesive microspheres." Due to the high surface-to-volume ratio, much more intimate contact with the mucus layer, and the precise targeting of drugs at the absorption site, Mucoadhesive microspheres provide advantages such as efficient absorption and improved bioavailability of drugs. 6

\section{APPROACHES TO FABRICATE GASTRO- RETENSIVE SYSTEMS}

- Floating or Low-density delivery systems

- Swelling systems

- High-density systems

- Bioadhesive or Mucoadhesive systems 


\section{MUCOADHESION}

Mucoadhesive drug delivery systems are used to improve drug absorption at appropriate sites. [6] Mucoadhesive is characterized as a process in which two materials, at least one of which is biological in nature, are kept together by interfacial forces over a long period of time. ${ }^{7}$

\section{ADVANTAGES OF MUCOADHESIVE DRUG DELIVERY SYSTEM}

$>$ The formulation at the delivery site stays longer, due to the adhesion and intimate contact, improving the bioavailability of the API using a lower API concentration in the treatment of disease. ${ }^{8}$

$>$ Better compliance with patients and convenience because of lower doses of drugs. ${ }^{8}$

$>$ It offers an excellent route for the systemic delivery of drugs with a high level of first-pass metabolism by offering higher bioavailability. ${ }^{9}$

$>$ Significant cost reductions achieved and dose-related side effects may be reduced due to the localization of the API at the disease site. ${ }^{10}$

$>$ Approximately the morphology of microspheres allows for controllable variability in degradation and release of drugs. 11

$>$ Sufficient and prolonged therapeutic effect. 11

The use of Mucoadhesive microspheres in the development of oral controlled drug delivery systems for effective gastrointestinal retention and sustained action. The Mucoadhesive system has proven to be very effective.

\section{TYPES OF MUCOADHESIVE POLYMERS 9, 10}

\section{First Generation Mucoadhesive Polymers:}

First generation Mucoadhesive polymers can be categorized into three major subcategories, particularly regarding:

1. Anionic polymers

2. Cationic polymers

3. Non-ionic polymers.

The strongest Mucoadhesive strength has been found among these anionic and cationic polymers. ${ }^{12}$

\section{Anionic polymers}

Due to their high Mucoadhesive functions and low toxicity, anionic polymers are the most often used as Mucoadhesive polymers in pharmaceutical formulations. These include alginates, carrageenan, and polyacrylic acid (PAA) and are weakly cross-linked with sodium-carboxymethylcellulose derivatives (NaCMC). Because of the high hydrogen-bonding interactions with mucin, PAA and NaCMC have excellent Mucoadhesive characteristics. 18 The derivatives of polycarbophil and carbomers (Carbopol and PAA), as a Mucoadhesive platform for drug delivery to the GI system, were extensively studied. ${ }^{7}$

\section{Cationic polymer}

Chitosan, a cationic polysaccharide similar to cellulose, is the most abundant polysaccharide on the globe. Chitosan is the most widely explored mucoadhesive polymer due to its biocompatibility, biodegradability, and favorable toxicological effects, and is becoming increasingly consequential. The linearity of Chitosan molecules also gives adequate chain interpenetration flexibility. Chitosan can offer enhanced drug delivery through the Mucoadhesive mechanism; drug absorption through paracellular routes has also been shown to improve by neutralizing fixed anionic sites within tight interlinked aligns between mucosal cells. ${ }^{13,14}$

\section{Novel Second-Generation Mucoadhesive polymers}

The second generation includes lectins and thiolated polymers:

\section{Lectins}

Non-immune proteins or glycoprotein complexes that can bind sugars selectively in a non-covalent manner are referred to as these. Lectins, which can bind to carbohydrates on the mucus or epithelial cell surface, have been studied extensively, especially for drug-targeting applications. These second-generation Bioadhesives are capable of not only cellular binding but also endocytosis and transcytosis. ${ }^{15}$

\section{Thiolated polymers}

These are also known as thiomers, which are hydrophilic macromolecules with free thiol groups on the backbone of the polymer. Morphological characteristics of polyacrylates and cellulose derivatives were greatly improved as a result of these functional groups. Since the polymer contains thiol groups, it can form stable covalent bonds with cysteine-rich sub domains of mucus glycoprotein's, resulting in increased residence period and enhanced bioavailability. Improved tensile strength, rapid swelling, and water absorption activity are some of the other beneficial Mucoadhesive properties of Thiolated polymers. Chitosan-thioglycolic acid, chitosan-thioethyl amidine, and alginate-cysteine are examples of Thiolated polymers. 16

\section{CLASSIFICATION OF MUCOADHESIVE POLYMERS}

There are various mucoadhesive polymers of synthetic and natural origin, which are classified in the Table below:

Table: A shortlist of Mucoadhesive Polymers 17

\begin{tabular}{|l|l|l|}
\hline $\begin{array}{l}\text { Sr. } \\
\text { No. }\end{array}$ & Synthetic Polymers & $\begin{array}{l}\text { Natural } \\
\text { Polymers }\end{array}$ \\
\hline 1. & $\begin{array}{l}\text { Hydroxypropyl methylcellulose } \\
\text { (HPMC) }\end{array}$ & Chitosan \\
\hline 2. & $\begin{array}{l}\text { Poly (acrylic acid) polymers } \\
\text { (carbomers, polycarbophil) }\end{array}$ & Sodium alginate \\
\hline 3. & Polyvinyl pyrrolidone (PVP) & Pectin \\
\hline 4. & Polyvinyl alcohol (PVA) & Locust Bean gum \\
\hline 5. & Polyhydroxyethyl methylacrylate & Guar gum \\
\hline 6. & Polyethylene oxide & Xanthan gum \\
\hline 7. & $\begin{array}{l}\text { Sodium carboxy methyl } \\
\text { cellulose (Na CMC) }\end{array}$ & Karaya gum \\
\hline 8. & Hydroxyl ethyl cellulose (HEC) & Gelatin \\
\hline 9. & Hydroxypropyl cellulose (HPC) & Tragacanth \\
\hline 10. & Ethyl cellulose (EC) & Soluble starch \\
\hline 11. & Methylcellulose (MC) & Lecithin \\
\hline
\end{tabular}




\section{MECHANISM OF MUCOADHESION 18, 19}

It includes 2 steps:

- The Contact Stage

- Consolidation Stage

\section{The Contact Stage}

The first phase of the Mucoadhesive Mechanism is the contact stage. This stage is marked by the association of the Mucoadhesive material with the mucosal surface, as well as by the swelling and spread of the formulation, which generates a deep contact with the mucosal surface.

\section{Consolidation Stage}

Mucoadhesive materials are activated in this process by the presence of moisture. The system is plasticized by humidity which allows the Mucoadhesive molecules to break free and link with weak hydrogen bonds.

\section{THEORIES OF MUCOADHESION 20, 21, 22}

\section{The Electronic Theory}

The electrons transfer between the Mucoadhesive polymer and the mucin glycoprotein network produces an electric double layer at the interface with the Mucoadhesive.

\section{The Wetting theory}

This signifies that when the contact angle of liquids is lower on the substrate surface, the liquid is more closely related to the substratum surface. In the presence of liquid, when two such substrates are brought into contact with each other, the liquid may act as an adhesive between the substrates.

\section{The Absorption theory}

According to this theory, the substance adheres to two surfaces after initial contact due to the surface force acting between the atoms in the two surfaces. Primary chemical bonds, which are covalent in nature, and secondary chemical bonds, which have a variety of different forces of attraction, such as electrostatic forces, Vander wall forces, hydrogen, and hydrophobic bonds, are two types of chemical bonds that arise from these forces.

\section{The Diffusion theory}

The polymer chains and the mucus mix are, in accordance with this theory, sufficient to create a semi-perpetuating adhesive bond. The diffusion coefficient and the time of contact determine the exact depth to which the polymer chain penetrates the mucus.

\section{The Mechanical Theory}

Explains how liquid adhesives diffuse through micro-cracks and irregularities on the Mucoadhesive substrate, resulting in Mucoadhesion and the creation of an interlocked structure.

\section{The Cohesive Theory}

According to this theory, Mucoadhesion is primarily caused by intermolecular interactions between like-molecules.

\section{EVALUATION PARAMETER OF MUCOADHESIVE MICROSPHER}

\section{Particle Size and Shape}

Microspheres' size, shape, and outer structure can be determined using light microscopy (LM) and scanning electron microscopy (SEM). ${ }^{23}$

\section{Entrapment Efficiency}

Allowing washed microspheres to lyse can be used to calculate the microspheres' entrapment efficiency or percent entrapment. The lysate is then filtered or centrifuged, and the active constituents are determined according to the monograph's standards. ${ }^{24}$

\section{$\%$ Entrapment $=$ Actual content $/$ Theoretical content $\mathbf{x}$ 100}

\section{Swelling index}

It demonstrates the ability of Mucoadhesive microspheres to swell at the absorbing surface by absorbing fluids present at the absorption site, which is a prerequisite for Mucoadhesion to start. 25 The following equation can be used to calculate the percent swelling value.

\section{Percent swelling $=\mathrm{D}_{\mathrm{T}}-\mathrm{D}_{\mathbf{0}} / \mathrm{D}_{\mathbf{0}} \times \mathbf{1 0 0}$}

Where $\mathrm{D}_{0}=$ weight of dried microspheres

$\mathrm{D}_{\mathrm{T}}=$ weight of swelled microspheres

\section{Surface Charge Study}

Photon correlation spectroscopy data can be used to measure the surface charge (zeta potential) of Mucoadhesive microspheres. The surface charge can be calculated using in-built software that uses the HelmholtzSmoluchowski equation to convert measured electrophoretic mobility to zeta potential. ${ }^{26}$ The zeta potential is a particle surface charge predictor that can be used to predict and monitor Mucoadhesion adhesive strength, stability, and mechanisms. Mucoadhesion is a mechanism in which mucus interacts with Mucoadhesive polymers, which is affected by their structure, including charge etc.

\section{In- Vitro Release Study}

Using a rotating basket or paddle-type dissolution apparatus, standard IP/BP/USP dissolution apparatus is used to research in-vitro release profiles in dissolution media that is close to the fluid present at the absorption site as per monograph. 28

\section{METHODS OF PREPARATION OF MUCOADHESIVE MICROSPHERES}

Mucoadhesive microspheres can be prepared by using different techniques like:

$\begin{array}{ll}\text { 1. } & \text { Complex Coacervation } \\ \text { 2. } & \text { Hot melt microencapsulation } \\ \text { 3. } & \text { Single emulsion technique } \\ \text { 4. } & \text { Double emulsion method } \\ \text { 5. } & \text { Solvent Evaporation Method } \\ 6 . & \text { Ionotropic gelation Method } \\ \text { 7. } & \text { Phase inversion method } \\ \text { 8. } & \text { Spray drying Method }\end{array}$

\section{Complex Coacervation 29, 30}

The theory of this approach is that when two hydrophilic colloids' solutions are combined under appropriate conditions, the liquid precipitate separates. The coating material process, made by dissolving an immiscible polymer in a suitable vehicle, and the core material are dispersed in a coating polymer solution under constant stirring in this system. Changing the temperature of the polymer solution; changing the $\mathrm{pH}$ of the medium; applying salt or an incompatible polymer or a non-solvent to the polymer solution; inducing a polymer-polymer interaction were all 
used to achieve microencapsulation. To shape a selfsustaining microsphere, the coating is typically hardened using thermal cross-linking or desolation techniques.

\section{Hot Melt Microencapsulation 31, 32}

This method was used to create microspheres of Polyanhydride copolymer of poly bis (p-carboxyl phenoxy) propane anhydride with sebacic acid. The polymer is melted first, and then the solid drug particles are added in a continuous mixing process. To obtain a stabilized emulsion, the prepared mixture is suspended in a non-miscible solvent such as silicone oil and heated at a temperature above the melting point of the polymer with continuous stirring. After cooling the emulsion to solidify the polymer particles, the microspheres are filtered and washed with petroleum ether.

\section{Double Emulsion Method 33, 34]}

This method was first defined by Ogawa Y et al. in 1988, and it is the most commonly used microencapsulation method today. To make a primary water-in-oil emulsion, an aqueous solution of drug and polymer is applied to the organic process with vigorous stirring. To obtain several emulsions $(\mathrm{w} / \mathrm{o} / \mathrm{w})$, this emulsion was poured into a large volume of water containing an emulsifier such as polyvinyl alcohol or polyvinylpyrrolidone and stirred until most of the organic solvent evaporated, leaving stable microspheres. After that, the microspheres are cleaned and dried.

\section{Solvent Evaporation 35, 36}

This is done in a liquid production vehicle. The microcapsule coating is dispersed in a volatile solvent that is incompatible with the liquid manufacturing vehicle phase of the process. In the coating polymer solution, a central substance to be microencapsulated is dissolved or dispersed. The core material mixture is distributed in the liquid manufacturing vehicle process with agitation to achieve the desired microcapsule size. If required, the mixture is heated to evaporate the solvent from the core material's polymer, after which the polymer shrinks around the core. Matrix-type microcapsules are created when the core material is dissolved in the coating polymer solution.

\section{Ionotropic Gelation Method 37, 38}

The drug is added to the aqueous sodium alginate solution by this method. To obtain a complete solution, the stirring continues and, after that, the solution containing $\mathrm{Ca} 2+/ \mathrm{Al} 3+$ is added drop-wise. The microspheres formed were kept in the original solution for 24 hours for internal gellification followed by filtration for separation. The total release is achieved at $\mathrm{pH}$ 6.4-7.4, but the drug will not release at acidic $\mathrm{pH}$. There are two methods by which microspheres can be generated using the ionotropic gelation technique, the external ionotropic gelation/cross-linking method, and the internal ionotropic gelation/emulsification method.

\section{Phase Inversion Method 39}

The method includes the addition of a drug in a diluted polymeric solution and is poured into an unstirred, petroleum ether bath in the ratio of 1:100 in methylene chloride. The production of microspheres is then clarified, petroleum ether washed and air-dried.

\section{Spray Drying Method 40}

This process entails dissolving/dispersing the drug into a polymer solution then spray-drying it. By this method, the size of the microspheres can be controlled by the manipulation of the spraying rate, the feed rate of the ISSN: 2250-1177 polymer-drug solution, the size of the nozzle, and the drying temperature.

\section{DRUG LOADING IN MICROSPHERES 41}

The drugs are loaded into the microspheres in one of two ways: during the microsphere preparation process or after the microsphere preparation process by incubating them with the drug solution. Physical entrapment, chemical bonding, and surface absorption are all strategies for the loading of active components. It was discovered that the addition of the drug during the preparation phase would achieve the highest loading of the drug in the microspheres, but this could be affected by a number of other process variables, such as the presence of additives, the preparation method, the polymerization heat, the agitation intensity, and so on. After preparing the microspheres, the drug can be loaded by incubating them in a suitable solvent with a high concentration of the drug. A Drug may be loaded into microspheres by penetration or diffusion of the drug through the microsphere's pores, as well as absorption of the drug on the microsphere's surface. After that, the solvent is extracted, leaving drug-loaded microspheres behind.

\section{FACTORS AFFECTING MUCOADHESION 43}

The Mucoadhesion of a drug carrier system to the mucous membrane depends on the below- mentioned factors:

\section{$>$ Polymer Based Factors}

- The molecular weight of the polymer

- Concentration of the polymer

- Stereochemistry of the polymer

- The chain length of the polymer

- Hydration of the polymer

\section{$>$ Physical Factors}

- $\mathrm{pH}$ at the polymer substrate interface

- Swelling of the polymer

- Applied strength

- Contact time

\section{APPLICATIONSOF MUCOADHESIVE MICROSPHERES 44}

\section{Oral drug delivery}

Microspheres containing polymer can form films, which allows them to be used in the formulation of film dosage forms as an alternative to pharmaceutical tablets.

\section{Gene delivery}

Because of their adhesive and transport properties in the GI tract (e.g., Chitosan, gelatin), microspheres may be a useful oral gene carrier.

\section{Nasal drug delivery}

Polymer-based drug delivery systems, such as microspheres, have been shown to have strong Bioadhesive properties and swell easily when in contact with the nasal mucosa, thus improving the drug's bioavailability and residence period.

\section{Buccal drug delivery}

Since it has Mucoadhesive or Bioadhesive properties and can serve as an absorption enhancer, the polymer is an excellent choice for buccal distribution.

Mucoadhesive microspheres are used as targeted drug delivery system for various diseases Such as: 
$>$ Hypertension

$>$ Diabetes mellitus

$>$ Peptic ulcer

$>$ AIDS

\section{CONCLUSION}

The phenomenon of Mucoadhesion can be used for several drug candidates as a model for controlled drug delivery approaches. Microspheres have a variety of benefits, including safety and masking, reduced dissolution rate, and specific targeting of the active ingredient. The Mucoadhesive microsphere's promising goal is to achieve controlled release with increased bioavailability and drug targeting to specific body sites.

\section{REFERENCES}

1. Galati UC, Chaudhary PD, Preparation Evaluation of Bioadhesive Microspheres Prepared by Ion Gelation Method and Effect of Variables on Quality of Microspheres. American Journal of Pharmtech. Research. 2013; 3(4):2249-3387.

2. Kadam NR and Suvarna V. Microspheres: A brief Review. Asian Journal of Biomedical and Pharmaceutical Sciences, 2015; 5(47):13-19.

3. Sachan AK, Gupta A, Arora M, Formulation \& characterization of nanostructured lipid carrier (NLC) based gel for topical delivery of etoricoxib, Journal of drug delivery and therapeutics 2016: 6(2):4-13

4. Parmar H, Bakliwal S, Gujarathi N, Rane B, Pawar S. Different method of formulation and evaluation of Mucoadhesive microsphere, International Journal of Applied Biology and Pharmaceutical Technology. 2010; 1(3):1157-1167.

5. Saisree R, Bhanja S, Das S, Verma B, Sudhakar M, Panigrahi BB. Formulation and Evaluation of Mucoadhesive Microspheres of Valsartan, Research Journal of Pharmacy and Technology.2019; 12(2):669-677.

6. Gavin PA, Laverty TP, Jones DS. Mucoadhesive Polymeric Platforms for Controlled Drug Delivery. European Journal of Pharmaceutics and Biopharmaceutics. 2009; 71: 505-518.

7. Deshmukh MT, Mohite SK. Preparation and Evaluation of Mucoadhesive Microspheres Fluoxetine $\mathrm{Hcl}$, International Journal of Pharmaceutical Sciences and Research. 2017; 8(9):3776-3785.

8. Shaikh R, Singh TRR, Garland MJ, Donnelly RF, Mucoadhesive Drug Delivery System, Journal of pharmacy and Bioallied Sciences, 2011; 3(1):89-100.

9. Punitha S, Girish Y. Polymers in Mucoadhesive buccal drug delivery system, International Journal of Research and Pharmaceutical Sciences. 2010; 1(2):170-186.

10. Parmar H, Bakliwal S, Gujarathi N, Rane B, Pawar S. Different methods of formulation and evaluation of Mucoadhesive microsphere, International Journal of Applied Biology and Pharmaceutical Technology. 2010; 1(3):0976-4550.

11. Kaurav H, Hari Kumar SL, Kaur A. Mucoadhesive Microspheres as carriers in Drug Delivery: A Review, International Journal of Drug Development \& Research. 2012; 4(2):0975-9344.

12. S. Kataria, Middha A, Sandhu P, Bilandi A and Kapoor B. Microsphere: A Review, International Journal of Research in Pharmacy and Chemistry. 2011; 1(4):1185-1198.

13. Ludwig A. The use of Mucoadhesive polymers in ocular drug delivery, Advanced Drug Delivery Reviews, 2005; 57(11):15951639.

14. Fefelova N, Nurkeeva Z, Mun G, Khutoryanskiy V. Mucoadhesive interactions of amphiphilic cationic copolymers based on [2 (methacryloyloxy) ethyl] trimethylammonium chloride, International Journal of Pharmacy, 2007; 339:25-32.

15. Khutoryanskiy VV. Hydrogen-bonded interpolymer complexes as materials for pharmaceutical applications, International Journal of Pharmaceutics. 2007; 334:15-26.

16. El-Kamel A, Sokar M, Naggar V, Al-Gamal S. Chitosan and sodium alginate-based bio adhesive vaginal tablets, American Association of Pharmaceutical sciences. 2002; 4:40-44.
17. Bravo-Osuna I, Vauthier C, Farabollini A, Palmieri GF, Ponchel G. Mucoadhesion mechanism of Chitosan and thiolated chitosanpoly (isobutyl cyanoacrylate) core-shell Nanoparticles, Biomaterials, 2007; 28(13):2233-2243.

18. Muthukumaran M, Dhachinamoorthi D, Chandra KBS, Sriram NA. Review On Polymers Used in Mucoadhesive Drug Delivery System. International Journal of Pharmacy and Industrial Research.2011; 1(2):122-127.

19. Hornof M, Weyenberg W, Ludwig A, Bernkop-Schnurch A. A Mucoadhesive ophthalmic insert based on thiolated poly (acrylic) acid: Development and in vivo evaluation in human volunteers, Journal of Controlled Release. 2003; 89:419-428.

20. Albrecht K, Zirm EJ, Palmberger TF, Schlocker W, BernkopSchnurch A, Preparation of thiomers micro particles and in vitro evaluation of parameters influencing their Mucoadhesive properties, Drug Development and Industrial Pharmacy. 2006; 32:1149-1157.

21. Punitha S, Girish Y. Polymers in mucoadhesive buccal drug delivery system: A review. International Journal of Research in Pharmaceutical Sciences. 2010; 1(2): 170-186.

22. Agusundaram M, Chetty MS, Umashankari K, Badrinath AV, Lavanya C, Ramkanth S. Microspheres as a novel drug delivery system: A review. International Journal of ChemTech Research. 2009; 1(3):526-534.

23. Carvalho FC, Bruschi ML, Evangelista RC, Gremial MPD. Mucoadhesive drug delivery systems. Brazilian Journal of pharmaceutical Sciences. 2010; 46: 1-17.

24. Harshad P, Sunil B, Nayan G. Different method of formulation and evaluation of mucoadhesive microsphere. International Journal of Applied Biology and Pharmaceutical Technology. 2010; 1(3):1157-1162.

25. Muthukumaran M, Dhachinamoorthi D, Chandra KBS, Sriram NA. Review on Polymers Used in Mucoadhesive Drug Delivery System. International Journal of Pharmacy and Industrial Research. 2011; 1(2):122-127.

26. Alagusundaram M, Chetty MS, Umashankari K, Badarinath AV, Lavanya C, Ramkanth S. Microspheres as a novel drug delivery system: A review, International Journal of ChemTech Research. 2009; 1(3):526-534.

27. Rajput G, Majmudar F, Patel J, Thakor R, Rajgor NB. Stomachspecific mucoadhesive microsphere as a controlled drug delivery system. Systematic Reviews in Pharmacy. 2010; 1(1):70-78.

28. Vyas TK, Babbar AK, Sharma RK, Singh S, Misra A. Intranasal mucoadhesive microemulsions of clonazepam: preliminary studies on brain targeting. International Journal of Pharmaceutical Sciences and Research. 2006; 95(3):570-580.

29. Bogataj M, Vovk T, Kerec M, Dimnik A, Grabnar I, Mrhar A. The correlation between zeta potential and mucoadhesion strength on pig vesical mucosa. Biological and Pharmaceutical Bulletin. 2003; 26(5):743 746 .

30. Sonani NG, Hiremath SP, Dasankoppa FS, Jama Kandi VG and Sreenivas SA. Design and evaluation of gastro protective Mucoadhesive cephalexin tablets. Pharmaceutical Development and Technology.2010; 15(2):178-183.

31. Muthukumaran M, Dhachinamoorthi D, Chandra KBS, Sriram NA Review on Polymers Used in Mucoadhesive Drug Delivery System. International Journal of Pharmacy and Industrial Research. 2011; 1(2):122-127.

32. Tarun V, Jyoti G. Pharmaceutical application of microspheres: An approach for the treatment of various diseases. International Journal of Pharmaceutical Science and Research. 2017; 8(8):3252 -3260.

33. Mathiowetz E and Langer R. Polyanhydride microspheres as drug carriers. Hot-Melt Micro-encapsulation Journal of Control Release. 1987; 5(1):13-22.

34. Garg A, Upadhyay P. Mucoadhesive Microspheres: A short review. Asian journal of pharmaceutical and clinical Research. 2012; 5(3):24-25.

35. Alagusundaram M, Chetty MS, Umashankari K, Badarinath AV, Lavanya C, Ramkanth S. Microspheres as a novel drug delivery system: A review. International Journal of ChemTech Research. 2009; 1(3):526-534

36. Bogataj M, Mrhar A and Korosec L. Influence of physicochemical and biological parameters on drug release from microspheres adhered on vesical and intestinal mucosa. International Journal of Pharmaceutics. 1999; 177(2):211-220. 
37. Parmar H, Bakliwal S, Gujarathi N, Rane B, Pawar S. Different method of formulation and evaluation of mucoadhesive microsphere. International Journal of Applied Biology and Pharmaceutical Technology. 2010; 1(3):1157-1167.

38. Kumari N, Aggarwal G, Harikumar S. L. Mucoadhesive microspheres: A review. Journal of Drug Delivery \& Therapeutics. 2014; 4(5):48-54.

39. Costa MS, Margarida Cardoso MM, Effect of uniform sized polymeric microspheres prepared by membrane emulsification technique on controlled release of anthracycline anti-cancer drugs, Desalination. 2006; 200:498-500.

40. Yassin AE, Alanazi FK, El-Badry M, Alsarra IA, Barakat NS Alanazi FK, Preparation and characterization of spironolactoneloaded gelucire micro particles using spray-drying technique,
Drug Development and Industrial Pharmacy2009; 35(3):297304 .

41. Zhang L, Liu Y, Wu Z and Chen H. Preparation and characterization of coacervate microcapsules for the delivery of antimicrobial oyster peptides. Drug Development and Industrial Pharmacy. 2009; 35(3):369-278.

42. Mathiowetz E, Kreitz MR and Peppas LB. Microencapsulation. In Mathiowitz E, ed. Encyclopedia of Controlled Drug Delivery, New York: John Willey \& Sons. 1999; 9: 493-504.

43. Moy A, Mathew S, Mathapan R, Prasanth V. Microsphere-An Overview. International Journal of Pharmaceutical and Biomedical Sciences. 2011; 2(2):322-338.

44. Shahina NS, Deepa M. Mucoadhesive Microspheres as a Drug Delivery System: A Review. Journal of Pharmaceutical Sciences and Research. 2019; 11(7):2734-2738. 